\title{
A reestruturação de um projeto odontológico para pessoas com TEA em tempos de
}

\section{Covid-19}

\author{
The Restructuring of a dental project for people with ASC in the Covid-19 pandemic \\ La reestructuración de un proyecto dental para personas con TEA en la pandemia Covid-19
}

Recebido: 09/12/2021 | Revisado: 14/12/2021 | Aceito: 18/12/2021 | Publicado: 02/01/2022

Fabiana Vargas Ferreira

ORCID: https://orcid.org/0000-0003-3567-4737

Universidade Federal de Minas Gerais, Brasil

E-mail: fabivfer@gmail.com

Fernanda Vargas Ferreira

ORCID: https://orcid.org/0000-0002-2009-3422 Universidade Federal do Pampa, Brasil

E-mail: anandafvf@gmail.com

Rosa Núbia Vieira de Moura

ORCID: https://orcid.org/0000-0002-8947-2797

Universidade Federal de Minas Gerais, Brasil

E-mail: bdmoura96@gmail.com

Juliana de Miranda Carrer

ORCID: https://orcid.org/0000-0003-1073-2021

Universidade Federal de Minas Gerais, Brasi

E-mail: jmcarrer@hotmail.com

Júlia Rodrigues Andrade

ORCID: https://orcid.org/0000-0003-2560-0216

Universidade Federal de Minas Gerais, Brasil

E-mail: julia.rodrigues.andrade@hotmail.com

Laura Gismonti de Araújo Gaudêncio

ORCID: https://orcid.org/0000-0003-2798-7960

Universidade Federal de Minas Gerais, Brasil

E-mail: lauragismonti@ufmg.br

Danielle Cristine Gonçalvez da Silva

ORCID: https://orcid.org/0000-0001-6752-4704

da Universidade Federal de Minas Gerais, Brasil

E-mail: daniellecrisgs@gmail.com

Dyorjenes Henrique Andrade Santos

ORCID: https://orcid.org/0000-0002-2780-3044

Universidade Federal de Minas Gerais, Brasil

E-mail: dyorjenes.andrade@gmail.com

Laura Beatriz de Jesus Souza

ORCID: https://orcid.org/0000-0003-3986-7595

Universidade Federal de Minas Gerais, Brasil E-mail: laurabeatrizjs@gmail.com

Lia Silva de Castilho

ORCID: https://orcid.org/0000-0001-9648-6815

Universidade Federal de Minas Gerais, Brasil E-mail: liasilvacastilho@gmail.com

\begin{abstract}
Resumo
Objetivo: este estudo descreve as estratégias adotadas por um Projeto de Extensão Odontológico para pessoas com Transtorno do Espectro Autista na Universidade Federal de Minas Gerais, abordando a introdução de mídias sociais e as tecnologias assistivas empregadas durante a pandemia do COVID 19 na interlocução com estes pacientes. Método: foi analisado o registro no SIEX deste projeto (403506) em relação aos resultados atingidos e aos produtos efetivamente entregues ao público alvo e à comunidade científica. Resultados: Foram encontrados vídeos institucionais e oficinas participativas demonstrando como elaborar materiais motivacionais para higiene bucal, alimentação e postagens em Instagram para a comunidade em geral e comunidade acadêmica, participações em eventos científicos, elaboração de cartilhas, redação de artigos científicos e dissertação de mestrado. Conclusão: A relação dialógica com a sociedade e o impacto e transformação social só poderão ser mensurados com o retorno presencial às clínicas. O emprego da comunicação remota pode ter prejudicado a interação entre a equipe multidisciplinar e está população tão singular em termos de interação social. O projeto de extensão "Atendimento
\end{abstract}


odontológico à pessoa com TEA" se esforçou em manter estes canais de relacionamento abertos com esta população e espera que este esforço possa mitigar os efeitos do isolamento social na promoção de saúde bucal da pessoa com TEA. Palavras-chave: Transtorno do Espectro Autista; COVID-19; Assistência odontológica para pessoas com deficiências; Educação à distância; Educação em odontologia.

\begin{abstract}
Objective: This study describes the strategies adopted by a Dental Outreach Project for people with Autistic Spectrum Disorder at the Federal University of Minas Gerais, addressing the introduction of social media and assistive technologies used during the COVID 19 pandemic in the dialogue with these patients. Method: the SIEX registration of this project (403506) was analyzed in relation to the results achieved and the products effectively delivered to the target audience and the scientific community. Results: Institutional videos and participatory workshops were found demonstrating how to develop motivational materials for oral hygiene, food and Instagram posts for the community in general and the academic community, participation in scientific events, preparation of booklets, writing of scientific articles and master's thesis. Conclusion: The dialogical relationship with society and the impact and social transformation can only be measured with the face-to-face return to the clinics. The use of remote communication may have impaired the interaction between the multidisciplinary team and this unique population in terms of social interaction. The extension project "Dental care for people with ASD" made an effort to keep these relationship channels open with this population and hopes that this effort can mitigate the effects of social isolation in promoting oral health for people with ASD.
\end{abstract}

Keywords: Autism Spectrum Disorder; COVID-19; Dental care for disabled; Education, distance; Education, dental.

\title{
Resumen
}

Objetivo: Este estudio describe las estrategias adoptadas por un Proyecto de Extensión Dental para personas con Trastorno del Espectro Autista en la Universidad Federal de Minas Gerais, abordando la introducción de las redes sociales y las tecnologías de asistencia utilizadas durante la pandemia de COVID 19 en el diálogo con estos pacientes. Método: se analizó el registro SIEX de este proyecto (403506) en relación a los resultados alcanzados y los productos efectivamente entregados al público objetivo y la comunidad científica. Resultados: Se encontraron videos institucionales y talleres participativos que demuestran cómo desarrollar materiales motivacionales para la higiene bucal, alimentación y publicaciones de Instagram para la comunidad en general y la comunidad académica, participación en eventos científicos, elaboración de folletos, redacción de artículos científicos y tesis de maestría. Conclusión: La relación dialógica con la sociedad y el impacto y transformación social solo se puede medir con el regreso presencial a las clínicas. El uso de la comunicación remota puede haber afectado la interacción entre el equipo multidisciplinario y esta población única en términos de interacción social. El proyecto de extensión "Atención odontológica para personas con TEA" hizo un esfuerzo por mantener abiertos estos canales de relación con esta población y espera que este esfuerzo pueda mitigar los efectos del aislamiento social en la promoción de la salud bucal de las personas con TEA.

Palabras clave: Trastorno del Espectro Autista; COVID-19; Atención dental para personas con discapacidades; Educación a distancia; Educación en odontología.

\section{Introdução}

O Transtorno do Espectro Autista é caracterizado por dois domínios que foram fortemente afetados pelo progresso da pandemia de COVID-19 no mundo O primeiro domínio é a dificuldade em interagir socialmente e dificuldade na comunicação para inciar ou responder a uma interação social, ajustando o seu comportamento para diversos contextos sociais e desenvolver e manter relações. O segundo domínio se caracteriza pela insistência em uniformidade e aderência incondicional às rotinas cuja quebra leva à uma profunda angústia em respostas a pequenas mudanças. Isso leva os pesquisadores a acreditarem que a vivência da pandemia por um indivíduo com TEA é única e talvez diversa daquela vivenciada por um indivíduo normotípico (Oomen et al., 2021).

A pandemia do COVID-19 levou a uma série de mudanças comportamentais com vistas ao seu enfrentamento dentro de bases de biossegurança. Essas mudanças envolvem o distanciamento social, ou mesmo um isolamento social rígido, uso de máscaras, lavagem frequente das mãos, higiene de superfícias rígidas, principalmente no período em que as vacinas ainda não estavam disponíveis (WHO, 2021). A vacinação em massa reduz drasticamente as hospitalizações pela síndrome respiratória aguda pelo Sars cov 2 (Barandalla et al., 2021). Mesmo assim, uma quarta onda da doença se inicia em 2021 levando países como a Alemanha a adotarem a comprovação de vacinação e exigindo o uso de máscaras para recintos fechados (Covid-19, 
2021).

Um grande dilema tem sido levantado pelos estudiosos dos Transtornos do Espectro Autista (TEA) durante a pandemia de COVID-19: as medidas de ansiedade estão agora refletindo uma característica de um indivíduo ou o estado de angústia intensificada resultante de lidar com o vírus? (Amaral et al., 2020).

Em um estudo conduzido na Malásia com indivíduos com TEA e normotípicos, a gravidade dos sintomas dos indivíduos com TEA não foi afetada comparando-se os períodos pré pandemia e período de lockdown. Entretanto, crianças com TEA tiveram maiores dificuldades de lidarem com a nova rotina quando comparados ao grupo controle. Crianças de ambos os grupos de diagnóstico também não demosntraram diferenças significativas. Em ambos os casos o nível de ansiedade ficou alto durante o bloqueio. As crianças de ambos os grupos não perceberam maiores sintomas de estresse e de depressão. Os autores ponderam que, algumas crianças se sentem mais confortáveis em casa do que com as tarefas e exercícios escolares. Pais de ambos os grupos relataram um aumento no estresse. Pais de indivíduos com TEA relatam maiores indices de depressão e diminuição do bem estar durante o período de confinamento do que pais de indivíduos normotípicos. Essas varíaveis foram relacionadas entre pais e filhos no grupo de indivíduos com TEA e não se relacionaram no grupo de indivíduos normotípicos (Fong et al., 2021). De fato, o estresse dos pais foi o preditor mais forte das percepções dos pais sobre o estresse infantil. Este resultado sugere que a saúde mental dos pais afeta a percepção e a resposta ao estresse e ansiedade do filho (Corbett et al., 2021; Vasa et al., 2021).

A nova pandemia da doença coronavírus (COVID-19) mudou a forma como a sociedade opera. Mudanças ambientais, rotinas interrompidas e acesso reduzido a serviços e redes sociais possuem um impacto único sobre os indivíduos com TEA e suas famílias e contribuíram para uma deterioração significativa em alguns. O acesso ao suporte é crucial para abordar fatores de vulnerabilidade, orientar ajustes em ambientes domésticos e aplicar estratégias de mitigação para melhorar lidar (Ameis et al., 2020).

Entre adultos, em um estudo conduzido na Bélgica, Holanda e Reino Unido, foram encontrados aumentos nos estados depressivos e de ansiedade em grupos de pessoas com TEA e indivíduos normotípicos. Entretanto, em adultos com TEA o aumento foi maior. As maiores preocupações se relacionavam com animais de estimação, trabalho, obtenção de medicamentos e alimentos, com sua prórpia segurança e com aperda de rotina. Um alívio no estresse resultante do convívio social foi sentido, embora a perda da interação social fosse reconhecida como algo difícil em suas vidas. Indivíduos com TEA sentiram um alívio ao perceberem a solidariedade demonstrada pelas demais pessoas e com a redução da sobrecarga sensorial e social. A interação social com pessoas usando máscaras foi um fator que dificulta a leitura das expressões faciais. Indivíduos com TEA também relataram necessitar de maiores informações específicas sobre sua condição com relação à pandemia. Os autores concluem que um suporte contínuo e específico dos serviços de saúde são imprescindíveis, focando na manutenção de uma rede social e no ajuste às rápidas mudanças na rotina exigidas (Oomen et al., 2021). O aumento da ansiedade e do estresse também foi notado entre adultos com TEA quando comparados com indivíduos típicos, no estudo condizido por Cobertt, Muscatello e Klemincic (2021) nos Estados Unidos durante o período de isolamento social.

A crise atual destaca que nossos sistemas de cuidados regulares não são suficientes para atender às necessidades de indivíduos com TEA em nossas comunidades. Em muitas partes do mundo, as pessoas passaram a frequentar a escola online e aumentaram o uso de dispositivos remotos para fornecimento de assistência médica e suporte para o TEA(Ameis et al., 2020).

O acesso a esses serviços precisa ser aumentado para mitigar os efeitos negativos do COVID-19 e futuras epidemias/pandemias. A rápida expansão no uso de plataformas de telessaúde tem um impacto positivo na assistência e na pesquisa. Além disso, pode ajudar a abordar as principais prioridades para as comunidades de indivíduos com TEA, incluindo longas listas de espera para avaliação e atendimento, acesso a serviços em locais remotos e horários restritos de serviço. No entanto, mudanças no sistema são urgentemente necessárias para garantir acesso equitativo e modelos de atendimento 
flexíveis, especialmente para famílias e indivíduos em desvantagem socioeconômica. A pandemia exige o uso de tecnologia para apoiar uma gama mais ampla de opções de cuidados e melhor atender às diversas necessidades das pessoas com TEA e suas famílias. Cabe a nós usar esta crise como uma oportunidade para promover a resiliência não apenas para um determinado indivíduo ou sua família, mas também o sistema: para conduzir mudanças duradouras e favoráveis ao autismo nos sistemas de saúde, sociais e contextos socioecológicos mais amplos (Ameis et al., 2020).

Oakley et al (2021) analisaram as políticas de saúde de 15 países membros da União Européia, publicadas entre março a julho de 2020 em relação ao acesso a testes diagnósticos para COVID-19, provisões para Unidades de Tratamento Intensiva (UTI) e as mudanças nos padrões de saúde e assistência social. Além disso, analisaram dados sobre as experiências vividas por 1301 pessoas com TEA e seus cuidadores. Os resultados destes estudo apontam que as pessoas com TEA tiveram inúmeras barreiras de acessos aos serviços de saúde durante a pandemia. A primeira barreira se refere à testagem e a segunda barreira se refere aos procedimentos de internação. Neste caso, muitos serviços de tratamento foram relatados como inacessíveis devido às diferenças individuais em relação às necessidades de comunicação. Em muitos países europeus, os protocolos de triagem para admissão em UTI's resultaram, diretamente ou indiretamente, em discriminação excludente sobre tratamentos que poderiam salvar vidas. Finalmente, cerca de 70\% de indivíduos com TEA ficaram sem suporte de diário de tratamentos de saúde e serviço social.

Todas essas modificações ambientais podem alterar a entrada sensorial (por exemplo, devido a ruídos mais altos ou diferentes em casa) e aumentar o estresse e a ansiedade entre pessoas com TEA e suas famílias (Ameis et al., 2020).

Contudo, esses desafios podem ser mitigados se o ambiente da pessoa em casa for confortável. Por exemplo, exposição a ambientes que causam sobrecarga social oudesconforto sensorial pode ser diminuído com o criterioso implementação de medidas de distanciamento físico exigidas pela pandemia. Com a reabertura gradual de escolas e negócios ocorrendo após a primeira onda de a pandemia em muitas partes do mundo, háo potencial de que as precauções serão reforçadas novamente para reduzir a propagação de vírus e sobrecarregar os sistemas médicos com ondas subsequentes. A necessidade de fazer a transição para frente e para trás será particularmente difícil para crianças e adultos autistas. Esta realidade significa que nossos sistemas de atendimento precisam permanecer aberto, acessível e flexível em resposta ao crise em evolução (Ameis et al., 2020).

A pandemia exacerbou ainda mais as desigualdades e a exclusão de pessoas com TEA, reduzindo a qualidade de vida, aumentando o sofrimento mental, as dificuldades comportamentais, a morbidade e a mortalidade pela COVID-19 (Oakley et al., 2021). Modelos de regressão indicam que os fatores de risco para o aumento de problemas psiquiátricos entre crianças com TEA incluem a compreensão da criança sobre a doença COVID-19, o adoecimento de algum familiar, baixa renda familiar e sintomas de depressão e ansiedade entre os pais da criança (Vasa et al., 2021).

No estudo de Vasa et al. (2021), em dois meses de isolamento social nos Estados Unidos, cerca de $60 \%$ de crianças com TEA ( $\mathrm{n}=257)$ tiveramaumento nos seus problemas psiquiátricos. Cerca de $45 \%$ da amostra teve um agravamento do seu problema psiquiátrico pre-existente e cerca de $50 \%$ apresentaram novos sintomas. Destes, apenas um terço não tinha problemas psiquiátricos pré-existentes. A depressão pré-existente era relativamente infrequente na amostra, mas foi o sintoma que mais se exacerbou, seguida por alterações comportamentais e transtornos de ansiedade.

O projeto de extensão Abordagem Multidisciplinar em Indivíduos com Transtorno do Espectro Autista (TEA) iniciou suas atividades no segundo semestre de 2019. O projeto contava com atividades práticas de atendimento odontológico primário às pessoas com TEA da Grande Belo Horizonte. Com a pandemia as atividades presenciais foram suspensas e novas estratégias para a promoção da relação dialógica com a sociedade foram elaboradas.

Desde a sua criação, o projeto previa a educação em saúde, prevenção e promoção de saúde por meio de redes sociais. No seu início, a previsão era a de que a realização das postagens seria realizada pelos alunos com supervisão dos docentes, 
sendo semanais e englobariam diversos temas.

Com a decisão da suspensão das atividades presenciais orientada pela Comissão do Enfrentamento à COVID-19 e Vigilância Sanitária da Prefeitura Municipal de Belo Horizonte, a equipe se reuniu para analisar quais seriam as alternativas possíveis para o cumprimento das diretrizes da Extensão universitária: relação dialógica com a sociedade; impacto e transformação social; impacto na formação do estudante; interprofissionalidade e indossociablidade ensino/pesquisa/extensão.

Tendo em vista o exposto, este artigo tem como objetivo descrever e analisar as propostas elaboradas pelo projeto de extensão Abordagem Multidisciplinar em Indivíduos com Transtorno do Espectro Autista (TEA) durante o período de isolamento social com base em evidências científicas. Com isso, espera-se auxiliar outros projetos semelhantes a construírem suas próprias estratégias de enfrentamento do problema da saúde bucal entre pessoas com TEA.

\section{2- Metodologia}

Este é um estudo de caso, observacional, descritivo que parte da análise documental de fonte direta dos registros de resultados alcançados e produtos entregues (Pereira et al., 2018) constantes na plataforma SIEX cujo número de identificação do referido projeto é: 403506. Além disso, foram analisados os registros de postagens no Instagram do projeto: @ odonto.autismo.ufmg. A análise descritiva se deu a partir da Resolução n 7, 18 de dezembro de 2018 (Brasil, 2018) que estabelece as diretrizes da Extensão Universitária.

\section{Resultados e Discussão}

Desde o seu início em 2019, participaram alunos do curso de Odontologia, como também dos cursos de Terapia Ocupacional, Psicologia, Medicina e Fisioterapia. Até dezembro de 2019 o projeto atendeu 13 crianças e adolescentes e suas respectivas famílias nas clínicas da Faculdade de Odontologia da UFMG. Como atividade de final de semestre realizou-se um encontro festivo denominado "Encontro Mundo Azul" que buscou estreitar as relações entre usuários, familiares, estudantes e professores do projeto. Foi uma oportunidade única para confraternização e acolhimento entre todos.

Com o advento da pandemia, este vínculo continuou através de postagens no Instagram do projeto@odonto.autismo.ufmg, uma vez que o quadro epidemiológico não permitiu mais atendimentos presenciais. No Instagram foram criados 7 personagens cujos nomes começam com as letras que compõem a palavra Autismo: Alex, Umberto, Thais, Isabela, Samuel, Mariana e Otávio. Cada personagem apresenta características específicas, incentivando a identificação com o público-alvo. As postagens, contendo notícias e confecção dos materiais para facilitar higiene bucal, por exemplo, são ofertadas no Instagram para pessoas com TEA, familiares, cuidadores, alunos e profissionais de saúde. Dentre essas postagens destacam-se a Cartilha sobre Direitos das Crianças e Adolescentes com TEA e a cartilha sobre uso de máscara, importância da vacinação e lavagem de mãos. Essas cartilhas foram elaboradas pelos estudantes do projeto. No Instagram também são postadas as Histórias Sociais. Atualmente, a História Social em desenvolvimento é sobre a ida à Faculdade de Odontologia, levando-se em conta as medidas de distanciamento e os protocolos de segurança. Também se trabalha com postagens focando em abordagem multidisciplinar para indivíduos com TEA.

Em agosto de 2021, o projeto teve a sua primeira defesa de Mestrado na área com o tema: Má oclusão em indivíduos com Transtorno do Espectro Autista, uma revisão sistemática e metanálise.

Em 2020, o projeto foi o representante do grupo Ciências da Vida- da UFMG na Associação de Universidades do Grupo Montevidéo (AUGM). Nessa apresentação toda a dinâmica em época de pandemia foi discutida com outros pesquisadores da área de indivíduos com necessidades especiais na América Latina. Além disso, o projeto, seus resultados e suas estratégias para estreitar os laços com os usuários e suas famílias foram apresentados em 18 eventos científicos nos anos de 2020 e 2021. Dentre esses eventos, a apresentação foi premiada como destaque na Jornada acadêmica da UFPI e na UCB. 
Desde agosto de 2020, este projeto de extensão faz parte do Programa de Atenção Interdisciplinar ao Autismo (PRAIA). Neste programa são concatenadas as ações das seguintes profissões de saúde: Psicologia, Medicina, Terapia Ocupacional e Odontologia. Ainda nesta época, uma professora da UNIPAMPA do curso de Fisioterapia se juntou ao grupo.

Finalmente, em outubro e novembro de 2021, o projeto apresentou duas oficinas sobre confecção de tecnologias assistivas para higiene bucal e educação para a saúde. Essas oficinas estão postadas no Instagram para acesso aos seguidores.

A pandemia afetou a sociedade tanto em relação ao adoecimento físico quanto no sofrimento psíquico. A saúde bucal foi negativamente impactada tanto em relação aos indivíduos normotípicos quanto entre os indivíduos com necessidades especiais. Os indivíduos com Transtorno do Espectro Autista formam um grupo peculiar, pois o estresse dos pais afeta diretamente o estresse dos filhos com TEA (Corbett et al., 2021; Vasa et al., 2021).

Desta forma, a ansiedade de pais e filhos tem potencial para causar mudanças de hábitos alimentares e de higienização bucal. Nesse aspecto, a diretriz da extensão universitária de relação dialógica com a sociedade (Brasil, 2018), talvez tenha sido uma das diretrizes mais importantes a se observar no contato remoto com indivíduos e responsáveis. Essa interação com o indivíduo não deve se focar apenas nos temas que orientam um projeto de extensão, no caso assistência odontológica.

Discutir com cuidadores e com os indivíduos sobre alimentação e higiene bucal é uma ação transformadora social tendo em vista a questão das preferências alimentares das pessoas com TEA: arroz, feijão, batata frita e bolachas (Moraes et al., 2021). Em relação à higiene bucal, o envolvimento dos cuidadores é fundamental também, pois o indivíduo pode não apresentar coordenação motora suficiente para uma satisfatória remoção do biofilme, mesmo quando dá o melhor de si (Amaral et al., 2016).

Trabalhar com equipes de outras profissões de saúde estende os limites da abordagem em saúde e ajuda na construção do conhecimento integrado, não fragmentado. Desta forma o público alvo compreende que a promoção de saúde é um conceito no qual aprender sobre saúde bucal, por exemplo, pode ajudar à melhoria da qualidade de vida em outros aspectos da saúde humana (Severo \& Seminotti, 2010). Mesmo no trabalho remoto, a interação entre diversos alunos dos cursos de saúde é rica em experiências exitosas (Castilho et al., 2020). Por isso, no presente projeto de extensão, a interlocução com outras profissões de saúde e a admissão de estudantes de outros cursos, enriquece a experiência do trabalho com extensão universitária.

O desenvolvimento de estudos científicos, em particular de uma Dissertação de mestrado, é a prova incontestável da indissociabilidade ensino/pesquisa/extensão. Apesar de ter sido uma metanálise, pois estudos epidemiológicos no momento não eram possíveis de serem realizados, o artigo redigido e submetido já se encontra em fase de adequação às exigências dos avaliadores. No período atual, a Universidade vem sofrendo tanto com as questões relacionadas à pandemia, como também em relação à questão de financiamento de pesquisas (Campos, 2020). O presente projeto também foi atingido por essas dificuldades.

Em relação ao impacto na formação do estudante, a possibilidade de desenvolver trabalhos científicos e divulgá-los em eventos nacionais e internacionais, inclusive se destacando, é uma oportunidade ímpar. Além disso, a experiência de confeccionar vídeos, cartilhas, histórias sociais sobre a saúde bucal e a pandemia servem para desenvolver o senso de empatia com o grupo de pessoas com Transtorno do Espectro Autista, cujo isolamento social se intensificou no período (Fong et al., 2021).

Sobre a metodologia empregada com o público alvo, podemos citar as Histórias Sociais. Essas histórias são adaptadas a partir do repertório comportamental de cada indivíduo com TEA. Seu objetivo é reforçar o comportamento desejado, no próprio ambiente em que o comportamento ocorre e precisa de um mediador (que no nosso caso é o cuidador) para alcançarem os seus objetivos (Silva, Arantes e Elias, 2020). Ao iniciarmos o trabalho com histórias sociais sobre como será o comportamento desejado no retorno às clínicas da FAO UFMG não podemos, por hora, aplicar o método no local de atendimento. Esperamos, contudo, que o comportamento seja introjetado e, no retorno, as histórias possam ser demonstradas 
nas clínicas. Aprender como elaborar uma História Social é um exemplo claro de interação entre os estudantes de diversos cursos. Este recurso não é facilmente dominado pelos estudantes de Odontologia nem pelas professoras. $\mathrm{O}$ trabalho junto aos estudantes de Psicologia proporcionou este diferencial.

Como o trabalho remoto também envolveu a participação de pais e cuidadores, a elaboração da cartilha foi um produto que envolveu estudantes na sua confecção. Discutir os direitos da pessoa com TEA com estudantes de outras áreas da saúde foi uma experiência transdisciplinar e inovadora. Com isso, pretende-se superar os limites das disciplinas através da reorganização estrutural do conhecimento a partir de vários pontos de vista (Jodelet, 2016) para os estudantes de Odontologia. Sem dúvida esse é um impacto na formação do estudante.

Os benefícios da realização de oficinas virtuais de expressão plástica e brincadeiras realizadas no Uruguai demonstram que esta modalidade à distância ajuda a mitigar os efeitos do isolamento social na continuidade aos processos terapêuticos grupais para crianças com TEA (Ávila et al., 2021). Essa foi a meta que os participantes do projeto buscaram ao desenvolverem as oficinas sobre construção de tecnologias assistivas para a higiene bucal de pessoas com TEA e com deficiência em geral.

Rossi et al. (2018) ao estudarem o acesso aos serviços de saúde por pessoas com TEA a partir da Análise de Redes Sociais concluem que a rede virtual de informações sobre atendimento para TEA possui uma baixa densidade. Segundo Vaz (2009) densidade é o percentual de elos existentes em uma rede dentre o total de possíveis elos. De qualquer forma, essa baixa densidade é um dado esperado uma vez que seu objetivo é auxiliar no caminho e na destinação dos serviços de saúde. Se a rede de informação estivesse incorporada a uma ação do governo, seria possível esperar maior geração de contatos e, por conseguinte, maiores difusão e dispersão de informações na rede, atingindo um público-alvo maior (Rossi et al., 2018). Para superar esse problema, o projeto trabalhou em conjunto com o grupo "Arte e Inclusão" em uma das oficinas e com a Próreitoria de Extensão em outra.

\section{Considerações Finais}

Ainda é cedo para estimarmos os impactos do isolamento social na promoção de saúde bucal de crianças e adolescentes com Transtorno do Espectro Autista. O isolamento social pode ter aliviado as tensões de enfrentamento dos ambientes para uma pessoa com TEA, mas também podem ter contribuído para a exclusão e solidão. Responder a estas questões é um objetivo para pesquisas futuras. Esta foi a experiência de um projeto de extensão pró-ativo que modificou seu rol de ações para o cumprimento de medidas sanitárias. De qualquer forma, entende-se que este foi o caminho possível de ser seguido. Em breve, o projeto poderá retornar de forma modesta e responsável.

\section{Referências}

Amaral, D. G., \& De Vries, P. J. (2020). COVID-19 and Autism Research: Perspectives from Around the Globe. Autism Research 13, $844-869$.

Amaral, L. D., Carvalho, T. F., \& Bezerra, A. C. B. (2016). Atenção bioética à vulnerabilidade dos autistas: A odontologia na estratégia da saúde da família. Revista Latinoamericana de Bioética, 16(1): 220-233

Ameis, S. H., Lai, M. C., Mulsant, B. H., \& Szatmari, P. (2020). Coping, fostering resilience, and driving care innovation for autistic people and their families during the COVID-19 pandemic and beyond. Molecular Autism 11:61. https://doi.org/10.1186/s13229-020-00365-y

Ávila, D. C., Macias, M. V., Mosca, B., Alvez, M., Hontou C., \& Larrosa, D. (2021). Oficinas terapêuticas para meninos e meninas com Transtorno do Espectro do Autismo: estratégias e possibilidades durante a pandemia de COVID-19. Estilos da Clínica, 26(2), 265-282. https://doi.org/10.11606/issn.19811624.v26i2 p265-282

Barandalla, I., Alvarez, C., Barreiro, P., Mendoza, C., González-Crespo, R., \& Soriano, V. (2021). Impact of scaling up SARS-CoV-2 vaccination on COVID19 hospitalizations in Spain. International Journal of Infectious Diseases 112 (1), 81-88. https://doi.org/10.1016/j.ijid.2021.09.022

Brasil. Ministério da Educação. Conselho Nacional de Educação. Câmara de Educação Superior. (2018). Resolução nº 7 , 18 de dezembro de 2018. Estabelece as Diretrizes para a Extensão na Educação Superior Brasileira e regimenta o disposto na Meta 12.7 da Lei $n^{\circ} 13.005 / 2014-2024$ e dá outras providências. http://portal.mec.gov.br/index.php?option=com_docman\&view=download\&alias=104251-rces007-18\&category_slug=dezembro-2018-pdf\&Itemid=30192. 
Campos, E. F. E. (2020). Ensino, pesquisa, extensão: Contribuições da pesquisa-ação. Revista Actualidades Investigativas em Educación, 20(1), 1-16. 10.15517/aie.v20i1.39972

Castilho, L. S., Vilaça, E. L., Leão, D. M., Moreira, L. D., Santos, B. P. R., \& Dias, D.R. (2020). Experience of a discipline of UFMG dentistry in crosscurricular training in accessibility and inclusion. Rev. Docência Ens. Sup. 10 (2), e023856. https://doi.org/10.35699/2237-5864.2020.23856

Corbett, B. A., Muscatello, R. A., \& Klemincic, M. E. (2021). The impactof COVID-19 on stress, anxiety, andcoping in youth with and without autism and their parents. AutismResearch. 14 (7):1496-1511. 10.1002/aur.2521.

Covid-19: Germany facing fourth wave and NI votes for mandatory Covid passports. Disponível em: https://www.bbc.com/news/uk-59322963

Fong, H. X., Cornish, K., Kirk, H., Ilias, K., Shaikh, M. F., \& Golden, K. J. (2021). Impact of COVID-19 lockdown in Malaysia: na examination of the psychological well-being of parent-child dyads and child behavior in families with children on the autistic spectrum. Frontiers on psychiatry.12. https://doi.org/10.3389/fpsyt.2021.733905

Jodelet, D. A representação: noção transversal, ferramenta da transdisciplinaridade. (2016). Cadernos de Pesquisa, 46, (162),1258-1271.

Moraes, L. S., Buboltz, V. K., Marques, A. C., Borges, L. R., Muniz, L.C., \& Bertacco, R. T. A. (2021). Seletividade alimentar em crianças e adolescentes com transtorno do espectro autista. R. Assoc. bras. Nutr. 12 (2), 42-58. https://doi.org/10.47320/rasbran.2021.1762

Oakley, B., Tilmann, J., Ruigrok, A., Baranger, A., Takow, C., Charman, T., Jones, E., Cusak, J., Dohert, M., Violland, P., Wroczynska A., Simonoff, E., Buitelaar, J. K., Gaççagher, L., \& Murphy, D. G. M. AIMS-2-TRIALS ECRAN \& the AIMS-2-TRIALS Consortium (2021). COVID-19 healthand social care access for autistic people: European policy review. BMJ Open 11:e045341. doi:10.1136/ bmjopen-2020-045341.

Oomen, D., Nijhof, A. D., \& Wiersema, J. R. (2021). The psychological impact of the COVID-19 pandemic on adults with autism: a survey study across three countries. Molecular Autism. 12:1-21. https://doi.org/10.1186/s13229-021-00424-y

Pereira, A. S., Shitsuka, D. M., Parreira, F. J., \& Shitsuka, R. (2018). Unidade 4- Metodologia de Estudo de caso. In: Metodologia da Pesquisa Científica. UFSM. Universidade Federal de Santa Maria. https://repositorio.ufsm.br/bitstream/handle/1/15824/Lic_Computacao_Metodologia-PesquisaCientifica.pdf?sequence $=1$

Rossi, L. P., Lovisi, G. M., Abelha, L., \& Gomide, M. (2018). Caminhos Virtuais e Autismo: acesso aos serviços de saúde na perspectiva da Análise de Redes Sociais. Ciência \& Saúde Coletiva, 23(10), 3319-3326

Severo, Silvani Botlender; SeminottI, Nedio. (2010). Integralidade e transdisciplinaridade em equipes multiprofissionais na saúde coletiva. Ciência \& Saúde Coletiva, 15 (Supl. 1), 1685-1698.

Silva, M. C., Arantes, A., \& Elias, N. C. Use of social stories in classroom to children with autism. (2020). Psicologia em Estudo, 25, e43094. 10.4025/psicolestud.v25i0.43094

Vasa, R. A., Singh, V., Holingue, C., Kalb, L., Jang, Y., \& Keefer, A. (2021). Psychiatric problems during the COVID-19 pandemic in children with autism spectrum disorder. Autism Res. 14(10):2113-2119. 10.1002/aur.2574.

Vaz, G. J. A construção dos sociogramas e a teoria dos grafos. (2009). Rev. Bras Psicodrama, 17(2):67-78. 